\title{
Brain Slump Caused by Jugular Venous Stenoses Treated by Stenting: A Hypothesis to Link Spontaneous Intracranial Hypotension with Idiopathic Intracranial Hypertension
}

\author{
${ }^{1}$ Department of Radiology, Addenbrooke's Hospital, Cambridge, \\ United Kingdom \\ ${ }^{2}$ Department of Neurosurgery, Addenbrooke's Hospital, Cambridge, \\ United Kingdom \\ ${ }^{3}$ National Hospital for Neurology and Neurosurgery, Queen Square, \\ London, United Kingdom \\ ${ }^{4}$ Department of Neurosurgery, Addenbrooke's Hospital, Cambridge, \\ United Kingdom
}

Nicholas Higgins ${ }^{1} \quad$ Rikin Trivedi $^{2} \quad$ Richard Greenwood $^{3}$ John Pickard ${ }^{4}$

J Neurol Surg Rep 2015;76:e188-e193.

\begin{abstract}
Address for correspondence Nicholas Higgins, FRCR, Department of Radiology, Addenbrooke's Hospital, Hills Road, Cambridge CB2 0QQ, United Kingdom (e-mail: nick.higgins@addenbrookes.nhs.uk).
\end{abstract}

Spontaneous intracranial hypotension, of which brain slump is an extreme expression, is caused by a cerebrospinal fluid leak. The reason the leak develops in the first place, however, is unknown, and some cases can be very difficult to manage. We describe a patient with severe symptoms of spontaneous intracranial hypotension and brain slump documented by magnetic resonance imaging whose clinical syndrome and structural brain anomaly resolved completely after treatment directed exclusively at improving cranial venous outflow. Diagnostics included computed tomography (CT) venography, catheter venography, and jugular venoplasty. CT venography showed narrowing of both internal jugular veins below the skull base. Catheter venography confirmed that these were associated with pressure gradients. Jugular venoplasty performed on two separate occasions as a clinical test gave temporary respite. Lasting remission (2 years of followup) was achieved by stenting the dominant internal jugular vein. These findings and this outcome suggest a mechanism for the development of spontaneous intracranial hypotension that would link it to idiopathic intracranial hypertension and have cranial venous outflow obstruction as the underlying cause.

\section{Introduction}

Idiopathic intracranial hypertension (IIH) is a syndrome of raised intracranial pressure of unknown cause, characterized by headache and visual symptoms, that can develop in either sex, at almost any age, with no obvious precipitating factor. ${ }^{1,2}$ Spontaneous intracranial hypotension is a condition of reduced intracranial pressure, characterized by orthostatic headache, brought about by the spontaneous development of a cerebrospinal fluid (CSF) leak. ${ }^{3}$

Both conditions are often discussed together, although more for reasons of aesthetic symmetry than for any known connection between them. Some patients presenting with spontaneous intracranial hypotension, however, can experience rebound intracranial hypertension after the CSF leak has been closed, suggesting that they might be related. ${ }^{4}$ received

January 16, 2015

accepted after revision

April 29, 2015

published online

June 12, 2015
DOI http://dx.doi.org/

10.1055/s-0035-1555015.

ISSN 2193-6366. (c) 2015 Georg Thieme Verlag KG

Stuttgart · New York
License terms

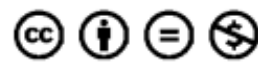




\section{Case Report}

A 55-year-old man was referred to clinic with a diagnosis of brain slump and probable CSF rhinorrhea. He had no complaint of headache, but his multiple symptoms included fatigue, low mood, loss of libido, poor memory, and difficulty concentrating. He was slow responding to questions and slow to grasp what was happening around him. His balance was poor. He was prone to dribbling, grunting, and abnormal movements of his face, head, and left arm. Clear fluid leaked intermittently from his right nostril.

His illness had developed insidiously $>3$ years earlier, characterized in the beginning by depression and fatigue. Symptoms then progressed after an incident in which he had fallen and hit the back of his head one night. He was not hospitalized at the time but had been unable to work since. The fluid leak from his nose had started at some unspecified time afterward.

Cardiac investigations had established no cause for blackouts. In neurology assessment the abnormal movements had been noted, but otherwise there were no abnormal clinical signs apart from bilateral extensor plantar responses. Routine blood tests had been unremarkable. CSF examination had been normal. Magnetic resonance imaging (MRI) had been reported as normal, and he was diagnosed with depression pending a repeat of his imaging.

Unexpectedly, the new MRI showed distortion and caudal displacement of the brainstem (-Fig. 1), an appearance in retrospect present on the previous scan, and, after specialist neuroradiological review, he was diagnosed with brain slump. Spinal MRI was normal. Computed tomography (CT) of the facial bones showed clear sinuses. CT cisternography was unremarkable. Nasal endoscopy (without fluorescein) was unremarkable. Therefore he was referred to our institution with suspected intracranial hypotension in the face of

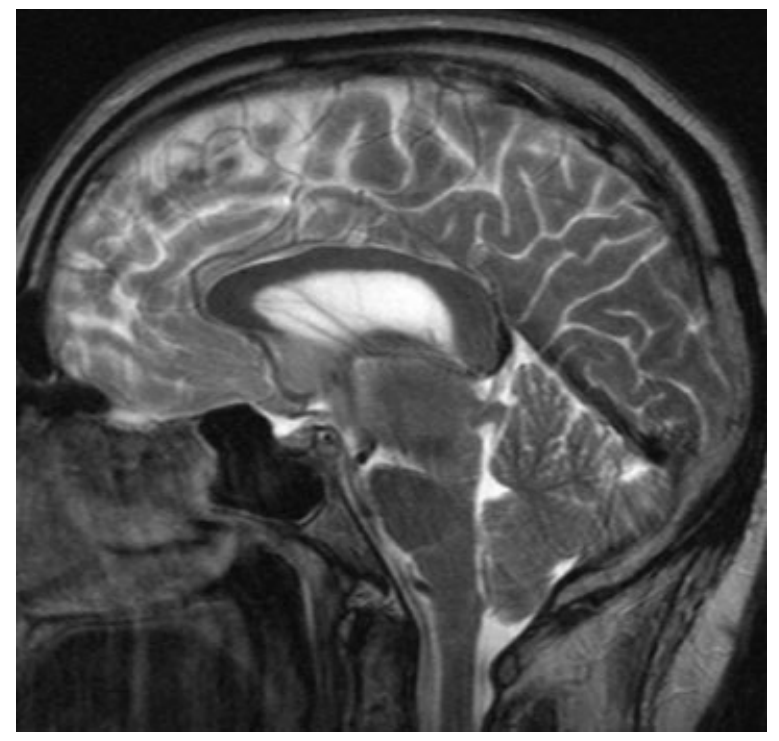

Fig. 1 Sagittal T2-weighted magnetic resonance image showing descent and distortion of the brainstem and obliteration of the basal cisterns. unremitting symptoms after the failure to prove, or find the source, of a CSF leak.

\section{Investigation of Intracranial Pressure}

CT venography showed normal intracranial venous sinuses but marked focal narrowing of both internal jugular veins below the skull base, between the styloid processes and lateral masses of $\mathrm{C} 1$ (-Fig. 2).

Catheter venography confirmed quite marked asymmetry of cranial venous outflow with the left side dominant. There was mild intracranial venous hypertension (mid-sagittal sinus pressure, $22 \mathrm{~cm} \mathrm{H}_{2} \mathrm{O}$ ) with a $5 \mathrm{~cm} \mathrm{H}_{2} \mathrm{O}$ pressure gradient between the mid-sagittal sinus and right atrium that included focal $3 \mathrm{~cm} \mathrm{H} \mathrm{H}_{2} \mathrm{O}$ pressure gradients across the jugular narrowings on each side.

Lumbar puncture gave an opening pressure of $18 \mathrm{~cm} \mathrm{H}_{2} \mathrm{O}$.

Balloon angioplasty of both internal jugular veins had no appreciable impact on venous anatomy at the time and brought about no immediate change to his condition. About 3 weeks later, however, his symptoms resolved abruptly, and he was able to return to work. Repeat MRI showed restoration of normal anatomy (-Fig. 3).

Four months later, following a long haul flight, his symptoms returned, and over a very short period he became as debilitated as before.

CT venography at this stage showed jugular venous anatomy similar to that when he was first seen, and sagittal reconstructions showed the return of brain slump. Repeat catheter venography revealed similar intravenous pressures. Repeat angioplasty, this time on the left side only, made no difference at first, but a few days later he reported an unequivocal improvement in symptoms. This lasted about 5 months before he reverted to his previous state when, once more, MRI showed brain slump (-Fig. 4A).

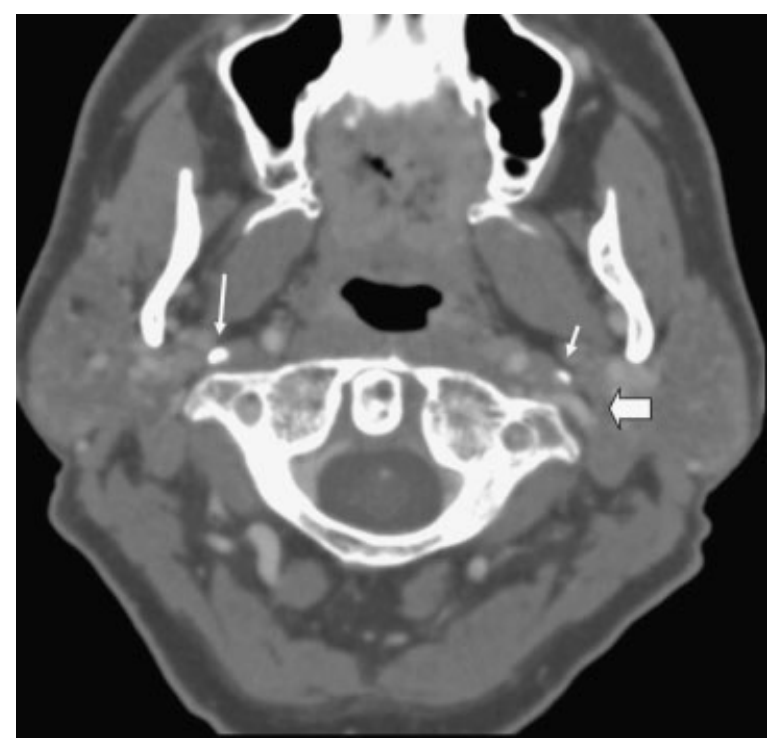

Fig. 2 Axial postcontrast computed tomography at the level of the $\mathrm{C} 1$ vertebra. The right jugular vein is rendered invisible behind the right styloid process (long arrow). The left jugular vein (large arrow) is less severely narrowed behind the left styloid process (short arrow). 


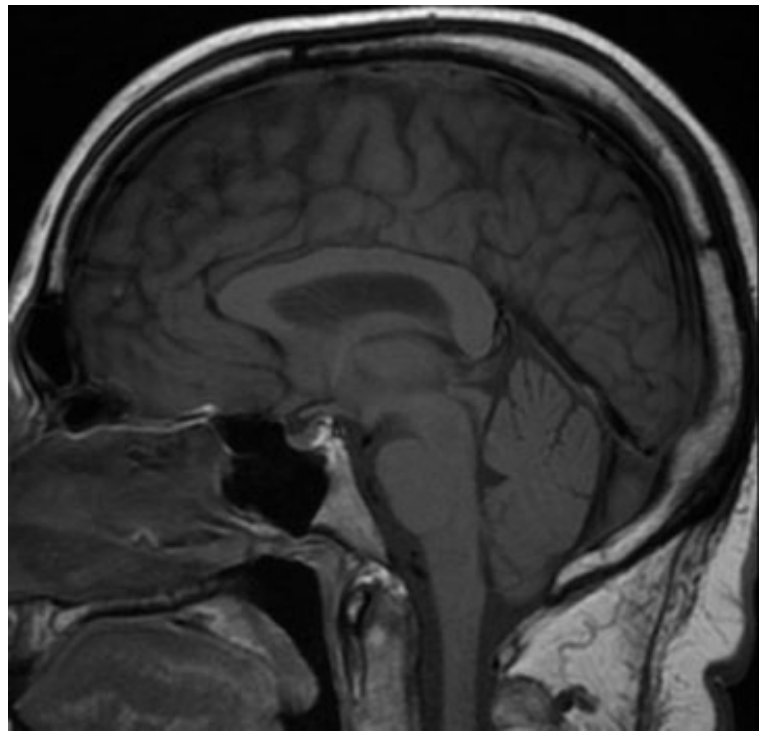

Fig. 3 Sagittal T1-weighted magnetic resonance image showing restoration of normal anatomy including of the basal cisterns, 3 weeks after bilateral jugular angioplasty.

\section{Jugular Venous Stenting}

Under general anesthesia two sheaths were placed in the left jugular vein to position two $5 \times 20$-mm self-expanding stents (Wallstent, Boston, Massachusetts, United States) side by side at the site of maximum narrowing. The reason for this particular configuration was to achieve the best possible expansion of the jugular vein within the constraints of the native anatomy at the $\mathrm{C} 1$ level where the vessel was flattened around the $\mathrm{C} 1$ lateral mass behind the styloid process (-Fig. 2). A longer third stent $(7 \times 60 \mathrm{~mm})$ was placed inside the lateral of the first two stents to give a shape that would lend stability to the stent complex by expanding into the jugular vein above and below the site of narrowing.

The final result was only achieved after several procedures. The first procedure, using a different stent configuration, was uneventful, but one of the stents had moved out of position by the next day. Because this was compromising the anatomical result, it was removed through a surgical venotomy. The remaining stent then thrombosed after surgery, and he had a further stenting procedure to the final configuration combined with direct thrombolysis.

Once a stable result was achieved, the fluid leak from his nose stopped, and he began to improve rapidly. He was discharged taking aspirin $75 \mathrm{mg}$ and clopidogrel $75 \mathrm{mg}$ daily as a precaution against further thrombotic complications. This was reduced to aspirin $75 \mathrm{mg}$ alone after 3 months. At 2month follow-up he was well, and MRI was normal (-Fig. 4B). - Fig. 5 shows the stents in situ.

\section{Progress and Follow-up}

Nine months after stenting, the patient had a partial recurrence of his symptoms. Catheter venography showed some intimal narrowing inside the stents ( - Fig. 6A, B), and sagittal CT reconstructions showed recurrence of brain slump. The narrowing was treated by balloon angioplasty ( - Fig. $\mathbf{6 C}, \mathbf{D}$ ) with no immediate change in his clinical condition. Four weeks later, however, he woke up feeling very well indeed, and repeat CT showed restoration of normal anatomy. He remains well, back in full employment, nearly 2 years following this last procedure.

\section{Discussion}

Distortion and caudal displacement of the brainstem and cerebellum, referred to as cerebellar or brain slump, are wellrecognized components of the syndrome of spontaneous intracranial hypotension, a condition characterized primarily by orthostatic headache in which CSF appears to be depleted by a spontaneous leak. ${ }^{3,5,6}$ Symptoms can be precipitated by minimal trauma, such as coughing or sneezing, presumably the stimulus for a sudden rise in intracranial pressure that ruptures the dura. What predisposes an individual to develop a dural breach under these circumstances, however, is unknown. ${ }^{3}$

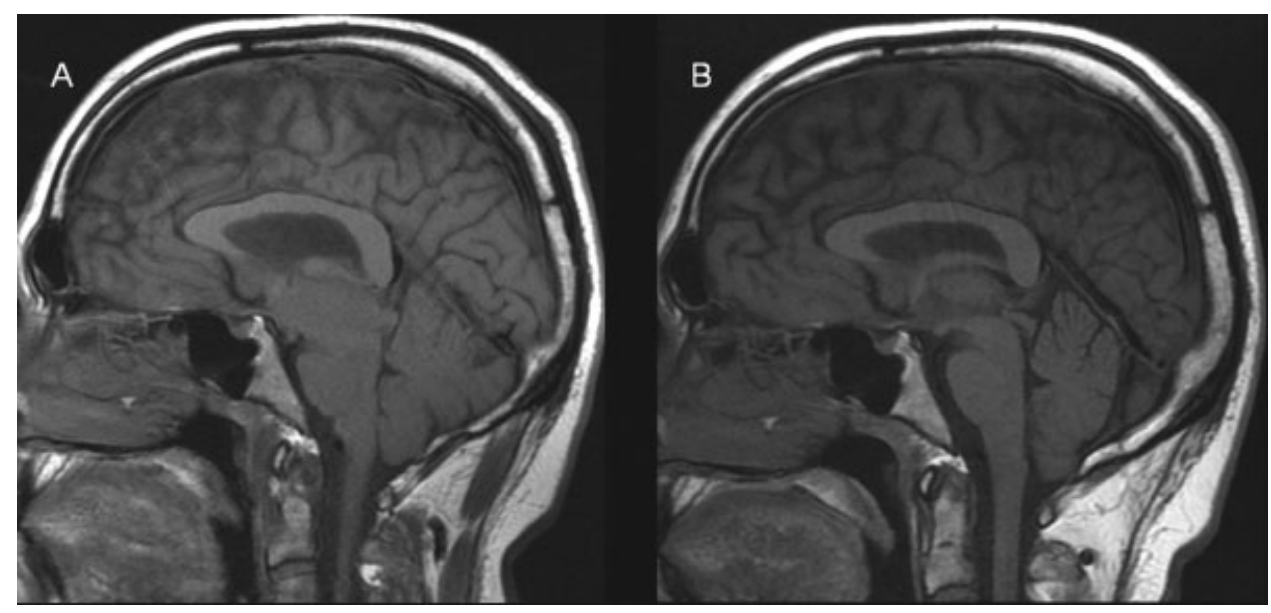

Fig. 4 Sagittal T1-weighted magnetic resonance image showing (A) recurrence of brain slump before left jugular stenting and (B) restoration of normal anatomical relationships afterward. 


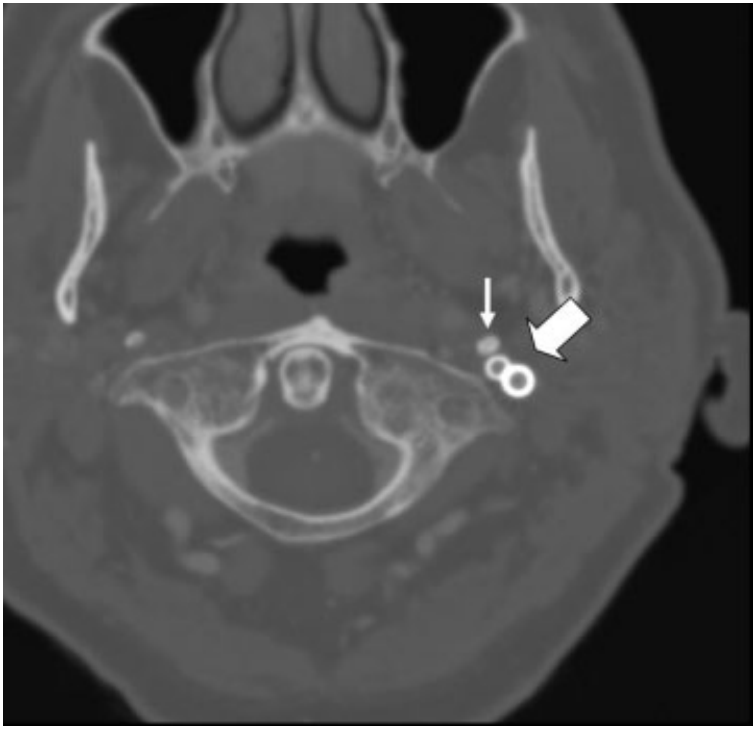

Fig. 5 Axial computed tomography of the neck at the $\mathrm{C} 1$ level showing the two stents (large arrow) in cross section, side by side, in front of the $\mathrm{C} 1$ left lateral mass and behind the styloid process (small arrow).

Symptoms are thought to arise from a loss of support for the brain in the cranium as CSF drains away in the upright posture, stretching pain-sensitive structures and distorting neural tissue. This produces one of the key features of the syndrome, a headache that develops on standing and is relieved by recumbency. Other symptoms, however, are usually present-nausea, vomiting, dizziness, visual disturbance, neck pain, for example-and may be severe. MRI usually shows characteristic appearances: caudal displacement of the hindbrain, small ventricles, subdural collections of fluid, and uniform thickening and enhancement of the dura. Lumbar puncture usually confirms low pressure. ${ }^{3,5,6}$

Treatment is generally conservative with most cases resolving over a period of weeks or months with little more than bed rest. When symptoms persist, investigations are aimed at locating the site of the presumed CSF leak with a view to sealing it. Treatment in the case of spinal leaks usually means an epidural injection of autologous blood., ${ }^{3,7}$ Treatment in the case of cranial leaks usually means surgical repair of the dural defect. ${ }^{8}$ Both treatments can be effective, a good clinical response essentially confirming the diagnosis as well as the site of CSF loss. In some cases, however, the source of the CSF leak can be difficult or impossible to find and symptoms very difficult to control. ${ }^{3,5,6}$

Some patients who appear to have the syndrome in all other respects do not complain of headache. Others have headache that is worse in recumbency, a phenomenon tentatively explained as related to dilatation of the cerebral veins and venous sinuses. ${ }^{9}$ Much more difficult to understand, however, are patients who appear to have the clinical syndrome but whose CSF pressures are in the normal range. ${ }^{9}$ This observation seriously questions current thinking on how the syndrome develops. It means that intracranial hypotension per se is not a requirement and, although not resolving the problem, has led to calls for spontaneous intracranial hypotension to be renamed as CSF depletion syndrome. ${ }^{10,11}$

The patient we describe is a case in point. Although headache was absent, his other symptoms were typical of intracranial hypotension, and the imaging confirmed marked slumping of the hindbrain, yet lumbar puncture revealed a CSF opening pressure of $18 \mathrm{~cm} \mathrm{H}_{2} \mathrm{O}$, that is, well within the normal range. So how is it that a patient can appear to have the symptoms and radiologic findings of intracranial hypotension, including a CSF leak, yet have a

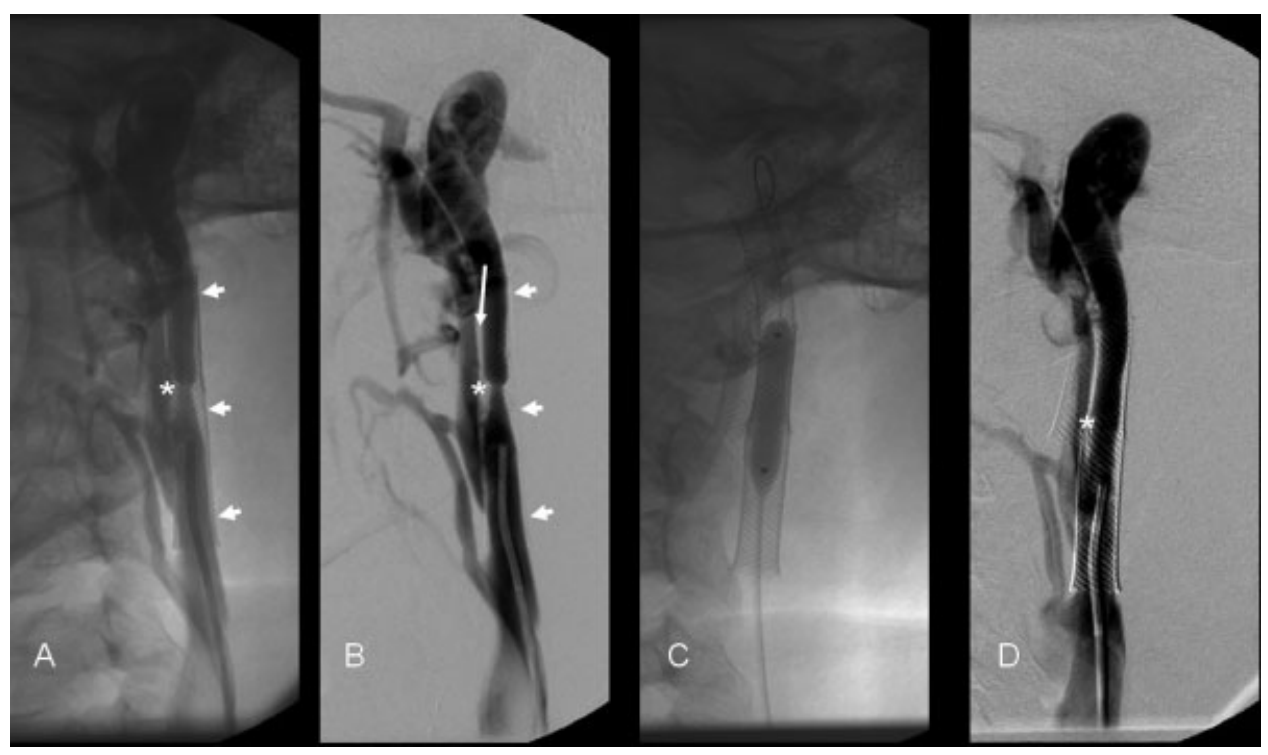

Fig. 6 Oblique frontal X-rays of the left neck, taken during angioplasty, 9 months after stenting. (A) Unsubtracted and (B) subtracted views showing focal narrowing of the lumen (asterisk) inside the lateral stent (small arrows). (C) Inflation of the balloon inside the stent. (D) Subtracted view following angioplasty showing resolution of in-stent stenosis (asterisk). 
normal CSF pressure? It is impossible to conceive that a CSF leak would do other than reduce CSF pressure. Therefore, these patients must have had CSF pressures in the premorbid state that were higher than those measured when they became symptomatic.

This raises the question of whether the premorbid CSF pressures in these patients, whatever they might have been, represent the normal physiologic state or pathology. If physiologic, the CSF leak would be the spontaneous event that precipitates the syndrome of CSF depletion. If pathologic, the CSF leak could be the consequence of abnormally raised CSF pressure, circumstances that would make CSF depletion syndrome one expression of an underlying disorder of raised intracranial pressure.

That CSF leaks can develop under conditions of raised intracranial pressure is evidenced by the condition of chronically raised intracranial pressure known as idiopathic intracranial hypertension (IIH). In this condition patients experience headaches and visual symptoms caused by the spontaneous development of raised intracranial pressure. ${ }^{1,2}$ Patients with IIH can develop or present with CSF leaks, with the assumption that high CSF pressures stress the dura until it gives way at a point of weakness. ${ }^{12,13}$ Evolution of the clinical syndrome subsequent to the leak then depends on the relative importance of the underlying IIH versus the effects of acquired CSF loss. Some patients with IIH, for example, describe an improvement in their usual headache coinciding with episodes of rhinorrhea.

This competition between two opposing forces acting on intracranial pressure is mirrored in the treatment of IIH by lumbar puncture or CSF diversion procedures, effectively iatrogenic CSF leaks. ${ }^{1,2}$ In these cases the benefit of reducing intracranial pressure by draining CSF has to be balanced against the deleterious effect of reducing CSF pressure too far. The predominant clinical expression of this latter phenomenon is orthostatic headache. ${ }^{14}$ Pertinent to this discussion is that some patients with IIH treated in this way develop orthostatic headaches even while CSF pressures are in the normal range. Indeed, such patients can very challenging to manage with symptoms that oscillate between high-pressure headaches, on the one hand, and orthostatic headaches, on the other as CSF pressures stray either side of a very narrow range of values.

These observations invite speculation that there may be a connection between IIH and CSF depletion syndrome, speculation that ought to be fueled by the knowledge that patients with IIH treated with lumboperitoneal shunts can acquire a Chiari-type deformity reminiscent of the brain slump that affects patients with CSF depletion. ${ }^{15,16}$ Thus one could postulate that the primary disorder in cases of CSF depletion syndrome, where the CSF pressure is normal, is likely one of raised intracranial pressure, and it would follow that the same should apply to many cases where CSF pressure is low. Moreover, because these patients generally show no abnormalities except for the presence of a leak and its effects, 3,5,6 this essentially means a diagnosis of IIH, IIH being a condition in which there are no clinical signs apart from those of raised intracranial pressure (signs which may be absent) and in which the radiology can be unremarkable. ${ }^{17}$ In this scenario, therefore, it should come as no surprise that successful treatment of a CSF leak can bring about overt intracranial hypertension indistinguishable from $\mathrm{IIH}^{4}$ although one would have to accept that any intracranial hypertension prior to the development of a leak was probably subclinical.

If IIH is the primary pathology underlying some cases of CSF depletion syndrome, discussion of the etiology of $\mathrm{IIH}$ becomes relevant to CSF depletion. This discussion generally focuses on four possible mechanisms: CSF overproduction, a failure of CSF absorption, brain swelling, and cranial venous outflow obstruction. None have been proven, but the difficulties alluded to previously in managing some patients with shunts argue against a problem in the CSF circulation. Intracranial venous sinus stenting could be said to address the concept of cranial venous outflow obstruction (and therefore, possibly, brain swelling), but there are no data, as yet, that prove that the intracranial venous narrowings that are a feature of IIH are the primary cause of raised intracranial pressure as opposed to being secondary to raised intracranial pressure itself. ${ }^{18}$

The possibility that extracranial venous obstruction might play a role in the etiology of IIH has not been addressed in any systematic way, although there is report of two cases in which IIH appeared to be caused by compression of the jugular veins between the styloid processes and lateral masses of the $\mathrm{C} 1$ vertebra. ${ }^{19}$ These cases are interesting because, like the patient we describe, they remove from consideration any effect that increased intracranial pressure itself might have on the venous narrowing, leaving the venous narrowing, unalloyed, as the cause of the clinical syndrome.

The case we describe, therefore, in which a patient with gross features of CSF depletion responded to treatment directed solely at improving cranial venous outflow, strongly suggests that obstruction to cranial venous outflow was at the root of the clinical problem. This suggests a hypothesis to explain the syndrome that at the same time would link it with IIH and would run as follows: cranial venous outflow obstruction, in one form or another, develops as the primary pathology. This drives increased intracranial pressure that, in turn, sets up the conditions for a CSF leak. The premorbid CSF pressure is pathologically raised but not enough to give symptoms that occasion medical intervention. The clinical syndrome that develops subsequent to the CSF leak is a conflation of the effects of venous obstruction tending to raise intracranial pressure and that of the CSF leak tending to reduce it. Relief of the venous obstruction by stenting results in a reduction in intracranial and CSF pressure that allows the dural defect to heal. The clinical syndrome resolves at a rate determined partly by the immediate effect of removing the venous obstruction and partly by the speed at which the CSF leak heals.

We therefore suggest that, in an unknown but possibly substantial number of cases, spontaneous intracranial hypotension/CSF depletion syndrome/brain slump is a manifestation of a disorder of raised intracranial pressure, essentially $\mathrm{IIH}$, and that cranial venous outflow obstruction is the underlying cause. 


\section{References}

1 Wall M, George D. Idiopathic intracranial hypertension. A prospective study of 50 patients. Brain 1991;114(Pt 1A):155-180

2 Sussman JD, Sarkies N, Pickard JD. Benign intracranial hypertension. Pseudotumour cerebri: idiopathic intracranial hypertension. Adv Tech Stand Neurosurg 1998;24:261-305

3 Mokri B. Spontaneous low pressure, low CSF volume headaches: spontaneous CSF leaks. Headache 2013;53(7):1034-1053

4 Mokri B. Intracranial hypertension after treatment of spontaneous cerebrospinal fluid leaks. Mayo Clin Proc 2002;77(11):1241-1246

5 Schievink WI, Deline CR. Headache secondary to intracranial hypotension. Curr Pain Headache Rep 2014;18(11):457

6 Urbach H. Intracranial hypotension: clinical presentation, imaging findings, and imaging-guided therapy. Curr Opin Neurol 2014; 27(4):414-424

7 Sencakova D, Mokri B, McClelland RL. The efficacy of epidural blood patch in spontaneous CSF leaks. Neurology 2001;57(10): 1921-1923

8 Sautter NB, Batra PS, Citardi MJ. Endoscopic management of sphenoid sinus cerebrospinal fluid leaks. Ann Otol Rhinol Laryngol 2008;117(1):32-39

9 Mokri B, Aksamit AJ, Atkinson JL. Paradoxical postural headaches in cerebrospinal fluid leaks. Cephalalgia 2004;24(10): 883-887

10 Mokri B. Spontaneous cerebrospinal fluid leaks: from intracranial hypotension to cerebrospinal fluid hypovolemia-evolution of a concept. Mayo Clin Proc 1999;74(11):1113-1123
11 Miyazawa K, Shiga Y, Hasegawa T, et al. CSF hypovolemia vs intracranial hypotension in "spontaneous intracranial hypotension syndrome”. Neurology 2003;60(6):941-947

12 Rosenfeld E, Dotan G, Kimchi TJ, Kesler A. Spontaneous cerebrospinal fluid otorrhea and rhinorrhea in idiopathic intracranial hypertension patients. J Neuroophthalmol 2013;33(2):113-116

13 Woodworth BA, Prince A, Chiu AG, et al. Spontaneous CSF leaks: a paradigm for definitive repair and management of intracranial hypertension. Otolaryngol Head Neck Surg 2008;138(6):715-720

14 Headache Classification Committee of the International Headache Society. The International Classification of Headache Disorders, 3rd edition (beta version). Cephalalgia 2013;33(9):629-808

15 Chumas PD, Armstrong DC, Drake JM, et al. Tonsillar herniation: the rule rather than the exception after lumboperitoneal shunting in the pediatric population. J Neurosurg 1993;78(4):568-573

16 Johnston I, Jacobson E, Besser M. The acquired Chiari malformation and syringomyelia following spinal CSF drainage: a study of incidence and management. Acta Neurochir (Wien) 1998; 140(5):417-427; discussion 427-428

17 Friedman DI, Jacobson DM. Diagnostic criteria for idiopathic intracranial hypertension. Neurology 2002;59(10):1492-1495

18 King JO, Mitchell PJ, Thomson KR, Tress BM. Manometry combined with cervical puncture in idiopathic intracranial hypertension. Neurology 2002;58(1):26-30

19 Dashti SR, Nakaji P, Hu YC, et al. Styloidogenic jugular venous compression syndrome: diagnosis and treatment: case report. Neurosurgery 2012;70(3):E795-E799 